\title{
Stress-tensor commutators in conformal field theories near the lightcone
}

\author{
Kuo-Wei Huang \\ Department of Physics, Boston University, Commonwealth Avenue, Boston, Massachusetts 02215, USA
}

(Received 8 July 2019; published 17 September 2019)

\begin{abstract}
Starting with the general stress-tensor commutation relations consistent with the Poincare algebra in local quantum field theory, we impose the tracelessness condition and focus on the dominating contributions in the lightcone limit. It is shown that, under a certain assumption on the Schwinger term, a Virasoro-algebra-like structure emerges near the lightcone in $d>2$ conformal field theories.
\end{abstract}

DOI: 10.1103/PhysRevD.100.061701

\section{INTRODUCTION}

As the universal central extension of the Witt algebra, the existence of the Virasoro algebra [1] plays a crucial role in mathematics and theoretical physics, particularly of deep importance in conformal field theory (CFT). It is, however, a special luxury one has in two-dimensional spacetime. In higher dimensions, where the conformal group is finite dimensional, Virasoro-algebra related techniques employed in understanding $d=2$ CFT become generally invalid. Nevertheless, one may still ask the following question: does an effective similar structure exist in higher-dimensional CFTs that can be used to control the CFT data within a certain subspace (i.e., a subsector of the full parameter space)?

As the form of two commuting copies, the Virasoro algebra can be expressed as the stress-tensor commutation relation

$$
\begin{aligned}
- & i\left[T^{++}\left(x^{-}\right), T^{++}\left(x^{\prime-}\right)\right] \\
= & 4\left(T^{++}\left(x^{-}\right)+T^{++}\left(x^{\prime-}\right)\right) \partial_{-} \delta\left(x^{-}-x^{\prime-}\right) \\
& -\frac{2 c}{3 \pi} \partial_{-}^{3} \delta\left(x^{-}-x^{\prime-}\right),
\end{aligned}
$$

with a similar expression for $\left[T^{--}\left(x^{+}\right), T^{--}\left(x^{\prime+}\right)\right]$. We denote $\left(x^{+}, x^{-}\right)=(t+y, t-y)$ with $\left(x^{0}, x^{1}\right)=(t, y)$, and $\left(T^{++}\left(x^{-}\right), T^{--}\left(x^{+}\right)\right)=-2\left(T_{0}^{0}-T_{1}^{0}, T_{0}^{0}+T_{1}^{0}\right) \quad$ in Minkowski metric $\eta_{\mu \nu}=\operatorname{diag}(-1,1)$. The central-extension part containing the central charge $c$ arises from the quantum anomaly. While the tracelessness condition in $d=2$ allows one to replace the purely spatial-component of the stress tensor, $T_{11}$, with $T_{00}$, independent spatialcomponents appear in $d>2$, and, in general, there is no

Published by the American Physical Society under the terms of the Creative Commons Attribution 4.0 International license. Further distribution of this work must maintain attribution to the author(s) and the published article's title, journal citation, and DOI. Funded by SCOAP ${ }^{3}$. stress-tensor algebra in higher dimensions. In this work, we make an initial attempt, starting from the most general stress-tensor commutation relations in Lorentz invariant, local quantum field theory (QFT) [2-4], to search for a possible Virasoro-like structure in higher-dimensional CFTs. In particular, while the tracelessness constraint must be imposed, we would like to see under what additional conditions an effective Virasoro-like algebra may emerge.

What clue do we have? The AdS/CFT correspondence [5-7] provides an interesting hint toward this direction. In a recent work [8], it was found that the operator product expansion (OPE) coefficients of the multi stress-tensor conformal blocks of a scalar four-point function in a large class of $d>2$ CFTs are universal in the lowest-twist subspace. (The twist of an operator is its dimension minus its spin.) These isolated OPE coefficients are universally fixed by the dimensions of the light and heavy scalar operators, and the central charge $C_{T}$, the coefficient of the stress-tensor two-point function. In $d=2$, the Virasoro algebra dictates all the related structures. While additional assumptions were made in the gravitational computation considered in [8], such as having a large $C_{T}$ and ignoring additional bulk matters, it is tempting to ask if the lowesttwist limit is essentially sufficient in a more general analysis in $d>2$ CFTs. As the lowest-twist limit corresponds to the lightcone limit, where operators in a correlator approach each other's lightcone, we are therefore motivated to consider the CFT stress-tensor commutators near the lightcone.

In the next section, we first review the general stresstensor equal-time commutation relations in QFT, based on earlier works [2-4]. The tracelessness condition and the lightcone limit shall be imposed in a later section. The main result is to obtain an effective lightcone stress-tensor commutator in CFT. By effective, we mean that the lightcone limit is taken when stress tensors are inserted in a correlation function. In this case, the purely lightcone component of the stress tensor, denoted as $\tilde{T}^{++}$below, dominates the contributions. A crucial point is that, in such an effective lightcone limit, one avoids the commutator 
with purely spatial components, i.e., $\left[T_{i j}(x), T_{m n}\left(x^{\prime}\right)\right]$, whose form cannot be determined by Poincaré symmetry or conformal invariance and thus is generally model dependent in $d>2$. (The central charge $C_{T}$ is also model dependent, but we say a quantity has a universal meaning if all the model-dependent data can be absorbed into $C_{T}$.) The resulting effective lightcone commutator will have a nonextension part and also a Schwinger term. The nonextension part formally looks the same as that in the $d=2$ Virasoro algebra. The Schwinger term in general dimensions can be related to the central charge $C_{T}$. Some subtleties of the Schwinger term will also be discussed.

\section{STRES-TENSOR COMMUTATION RELATIONS IN QFT}

Here we first review the stress-tensor commutators in Lorentz invariant, local QFT (see [4] and [2,3] for more discussions). Denote a classical action $S$ embedded in a curved background and write the curved-space stress tensor as

$$
C^{\mu \nu}(x)=2 \frac{\delta S}{\delta g_{\mu \nu}(x)} .
$$

A factor $\sqrt{-g}$ is normally factored out from $C_{\mu \nu}$ as the conventional stress tensor, but we adopt the above notation for later convenience. Eventually, we will be interested in the commutation relations of the flat-space stress tensor, denoted as $T^{\mu \nu}(x)$, in metric $d s^{2}=-d t^{2}+\delta_{i j} d x^{i} d x^{j}$. Below, we denote $\left\langle C^{\mu \nu}(x)\right\rangle=-2 i \frac{\delta Z}{\delta g_{\mu \nu}(x)}$, where $Z$ is the partition function. The starting point is to consider the variation of the conservation equation,

$$
0=\left\langle C_{; \nu}^{\mu \nu}(x)\right\rangle=\left\langle C_{, \nu}^{\mu \nu}(x)\right\rangle+\left\langle\Gamma_{\alpha \beta}^{\mu}(x) C^{\alpha \beta}(x)\right\rangle,
$$

with respect to an external metric. Varying the first term on the right-hand side of (3) yields

$2 \partial_{\nu} \frac{\delta\left\langle C^{\mu \nu}(x)\right\rangle}{\delta g_{\lambda \rho}\left(x^{\prime}\right)}=\partial_{\nu}\left(i\left\langle C^{\mu \nu}(x) C^{\lambda \rho}\left(x^{\prime}\right)\right\rangle_{+}+2\left\langle\frac{\delta C^{\mu \nu}(x)}{\delta g_{\lambda \rho}\left(x^{\prime}\right)}\right\rangle\right)$,

where $\left\langle C_{\mu \nu}(x) C_{\lambda \rho}\left(x^{\prime}\right)\right\rangle_{+}$is the time-ordered Lorentzian stress-tensor two-point correlator, while varying the second term on the right-hand side of (3) gives

$$
\begin{aligned}
2 \frac{\delta\left\langle\Gamma_{\alpha \beta}^{\mu}(x) C^{\alpha \beta}(x)\right\rangle}{\delta g_{\lambda \rho}\left(x^{\prime}\right)}= & \left(g^{\mu \lambda}\left\langle C^{\rho \alpha}(x)\right\rangle+g^{\mu \rho}\left\langle C^{\lambda \alpha}(x)\right\rangle\right. \\
& \left.-g^{\mu \alpha}\left\langle C^{\lambda \rho}(x)\right\rangle\right) \partial_{\alpha} \delta\left(x-x^{\prime}\right)+\mathcal{O}(\Gamma),
\end{aligned}
$$

where $\delta \Gamma_{\alpha \beta}^{\mu}=-g^{\mu \gamma} \delta g_{\gamma \sigma} \Gamma_{\alpha \beta}^{\sigma}+\frac{g^{\mu \gamma}}{2}\left(\partial_{\alpha} \delta g_{\gamma \beta}+\partial_{\beta} \delta g_{\gamma \alpha}-\partial_{\gamma} \delta g_{\alpha \beta}\right)$. The $\mathcal{O}(\Gamma)$ part vanishes in the flat-space limit. The above expressions lead to

$$
\begin{aligned}
i[ & \left.T^{\mu 0}(x), T^{\lambda \rho}\left(x^{\prime}\right)\right] \delta\left(x^{0}-x^{\prime 0}\right) \\
= & -\left.2 \partial_{\nu} \frac{\delta C^{\mu \nu}(x)}{\delta g_{\lambda \rho}\left(x^{\prime}\right)}\right|_{\text {flat }}-\left(\eta^{\mu \lambda} T^{\rho \alpha}(x)+\eta^{\mu \rho} T^{\lambda \alpha}(x)\right. \\
& \left.-\eta^{\mu \alpha} T^{\lambda \rho}(x)\right) \partial_{\alpha} \delta\left(x-x^{\prime}\right) .
\end{aligned}
$$

We have used $\partial_{\nu} C^{\mu \nu}(x)=\mathcal{O}(\Gamma), \quad\left\langle T^{\mu \nu}(x) T^{\lambda \rho}\left(x^{\prime}\right)\right\rangle_{+}=$ $\left\langle T^{\mu \nu}(x) T^{\lambda \rho}\left(x^{\prime}\right)\right\rangle \theta\left(x^{0}-x^{\prime 0}\right)+\left\langle T^{\lambda \rho}\left(x^{\prime}\right) T^{\mu \nu}(x)\right\rangle \theta\left(x^{\prime 0}-x^{0}\right)$, and $\partial_{\nu} \theta\left(x^{0}-x^{\prime 0}\right)=\delta_{\nu}^{0} \delta\left(x^{0}-x^{\prime 0}\right)$. Note the equal-time commutator should not have an explicit time derivative of $\delta\left(x^{0}-x^{\prime 0}\right)$, but the right-hand side of (5) presently looks like it has such a dependence. Consistency requires that the object $\partial_{\nu} \frac{\delta C^{\mu \nu}(x)}{\delta g_{\lambda \rho}\left(x^{\prime}\right)}$ provides a cancellation.

It can be instructive to derive explicitly the commutator involving only temporal components; other components can be obtained in a similar manner. From (5),

$$
\begin{aligned}
& i\left[T^{00}(x), T^{00}\left(x^{\prime}\right)\right] \delta\left(x^{0}-x^{\prime 0}\right) \\
& =-\left.2 \partial_{\nu} \frac{\delta C^{0 \nu}(x)}{\delta g_{00}\left(x^{\prime}\right)}\right|_{\text {flat }}+\left(2 T^{0 i}(x) \partial_{i}+T^{00}(x) \partial_{0}\right) \delta\left(x-x^{\prime}\right) .
\end{aligned}
$$

Defining a parametrization function $t\left(x, x^{\prime}\right)$ via

$$
\left.2 \frac{\delta C^{0 \nu}(x)}{\delta g_{00}\left(x^{\prime}\right)}\right|_{\text {flat }}=t^{0 \nu, 00}\left(x, x^{\prime}\right)-\eta^{\nu 0} T^{00}(x) \delta\left(x-x^{\prime}\right),
$$

the right-hand side of (6) can be written as

$$
-\partial_{\nu} t^{0 \nu, 00}\left(x, x^{\prime}\right)-\partial_{0} T^{00}(x) \delta\left(x-x^{\prime}\right)+2 T^{0 i}(x) \partial_{i} \delta\left(x-x^{\prime}\right),
$$

with no time-derivative on $\delta\left(x^{0}-x^{\prime 0}\right)$ left. A direct manipulation using $T^{0 i}\left(x^{\prime}\right) \partial_{i} \delta\left(x-x^{\prime}\right)=-\partial_{0} T^{00}(x) \delta\left(x-x^{\prime}\right)+$ $T^{0 i}(x) \partial_{i} \delta\left(x-x^{\prime}\right)$ gives

$$
\begin{aligned}
& i\left[T^{00}(x), T^{00}\left(x^{\prime}\right)\right] \delta\left(x^{0}-x^{\prime 0}\right) \\
& =\left(T^{0 i}(x)+T^{0 i}\left(x^{\prime}\right)\right) \partial_{i} \delta\left(x-x^{\prime}\right)-\partial_{\nu} t^{0 \nu, 00}\left(x, x^{\prime}\right) .
\end{aligned}
$$

Similarly, using (5), it is straightforward to consider other components. We now tabulate the various commutators as follows:

$$
\begin{aligned}
& i\left[T^{00}(x), T^{00}\left(x^{\prime}\right)\right] \delta_{t}=\left(T^{0 i}(x)+T^{0 i}\left(x^{\prime}\right)\right) \partial_{i} \delta\left(x-x^{\prime}\right) \\
& \quad-s^{0,00}\left(x, x^{\prime}\right), \\
& \quad i\left[T^{00}(x), T^{0 i}\left(x^{\prime}\right)\right] \delta_{t}=\left(T^{i j}(x)+T^{00}\left(x^{\prime}\right) \delta^{i j}\right) \partial_{j} \delta\left(x-x^{\prime}\right) \\
& \quad-s^{0,0 i}\left(x, x^{\prime}\right),
\end{aligned}
$$

$$
\begin{gathered}
i\left[T^{00}(x), T^{i j}\left(x^{\prime}\right)\right] \delta_{t}=\left(T^{0 i}\left(x^{\prime}\right) \partial^{j}+T^{0 j}\left(x^{\prime}\right) \partial^{i}\right. \\
\left.-\partial^{0} T^{i j}(x)\right) \delta\left(x-x^{\prime}\right)-s^{0, i j}\left(x, x^{\prime}\right),
\end{gathered}
$$




$$
\begin{aligned}
& i\left[T^{0 i}(x), T^{0 j}\left(x^{\prime}\right)\right] \delta_{t}=\left(T^{0 j}(x) \partial^{i}+T^{0 i}\left(x^{\prime}\right) \partial^{j}\right) \delta\left(x-x^{\prime}\right) \\
& -s^{i, 0 j}\left(x, x^{\prime}\right) \text {, } \\
& i\left[T^{0 i}(x), T^{j k}\left(x^{\prime}\right)\right] \delta_{t}=\left(\delta^{i m} T^{j k}(x)-\delta^{i j} T^{k m}\left(x^{\prime}\right)\right. \\
& \left.-\delta^{i k} T^{j m}\left(x^{\prime}\right)\right) \partial_{m} \delta\left(x-x^{\prime}\right)-s^{i, j k}\left(x, x^{\prime}\right),
\end{aligned}
$$

where $\delta_{t} \equiv \delta\left(x^{0}-x^{\prime 0}\right)$ and the Schwinger term is $s^{\mu, \lambda \rho} \equiv$ $\partial_{\nu} t^{\mu \nu, \lambda \rho}$ with

$$
\begin{gathered}
t^{0 \nu, 00}=\left.2 \frac{\delta C^{0 \nu}(x)}{\delta g_{00}\left(x^{\prime}\right)}\right|_{\text {flat }}+\eta^{\nu 0} T^{00}(x) \delta\left(x-x^{\prime}\right) \\
t^{0 \nu, 0 i}=\left.2 \frac{\delta C^{0 \nu}(x)}{\delta g_{0 i}\left(x^{\prime}\right)}\right|_{\text {flat }}+\eta^{\nu i} T^{00}(x) \delta\left(x-x^{\prime}\right) \\
t^{0 \nu, i j}=\left.2 \frac{\delta C^{0 \nu}(x)}{\delta g_{i j}\left(x^{\prime}\right)}\right|_{\text {flat }}+\left(\eta^{\nu j} T^{0 i}(x)\right. \\
\left.+\eta^{\nu i} T^{0 j}(x)-\eta^{0 \nu} T^{i j}(x)\right) \delta\left(x-x^{\prime}\right) \\
\left.t^{i \nu, 0 j=} 2 \frac{\delta C^{i \nu}(x)}{\delta g_{0 j}\left(x^{\prime}\right)}\right|_{\text {flat }}+\left(T^{0 i}(x) \eta^{\nu j}+T^{0 \nu}(x) \delta^{i j}\right) \delta\left(x-x^{\prime}\right) \\
t^{i \nu, j k}=\left.2 \frac{\delta C^{i \nu}(x)}{\delta g_{j k}\left(x^{\prime}\right)}\right|_{\text {flat }} \\
-\left(\delta^{i j} \eta^{\nu 0} T^{k 0}(x)+\delta^{i k} \eta^{\nu 0} T^{j 0}(x)\right) \delta\left(x-x^{\prime}\right)
\end{gathered}
$$

The commutation relation involving purely spatial components does not admit a model-independent form.

Some constraints on the Schwinger term must be imposed so that the above commutators remain consistent with the Poincaré relations

$$
\begin{aligned}
i\left[T^{\mu \nu}(x), P^{\lambda}\right]= & \partial^{\lambda} T^{\mu \nu}(x), \\
i\left[T^{\mu \nu}(x), J^{\lambda \sigma}\right]= & \left(x^{\lambda} \partial^{\sigma}-x^{\sigma} \partial^{\lambda}\right) T^{\mu \nu}(x)+\eta^{\mu \lambda} T^{\sigma \nu}(x)+\eta^{\nu \lambda} T^{\sigma \mu}(x) \\
& -\eta^{\mu \sigma} T^{\lambda \nu}(x)-\eta^{\nu \sigma} T^{\lambda \mu}(x),
\end{aligned}
$$

where $P^{\lambda}=\int d \vec{x} T^{0 \mu}, J^{\lambda \sigma}=\int d \vec{x}\left(x^{\lambda} T^{0 \sigma}-x^{\sigma} T^{0 \lambda}\right)$. One thus requires $\int \vec{d} \vec{x} s^{0,00}=\int d \vec{x} x^{i} s^{0,00}=0$. On the other hand, one may adopt $\bar{T}_{\mu \nu}=T_{\mu \nu}-\left\langle T_{\mu \nu}\right\rangle$, with $\left\langle T_{\mu \nu}\right\rangle \sim \eta_{\mu \nu}$, and check that the above structures remain formally the same. Below, we shall focus on flat-space CFT with a traceless stress tensor. As the expectation value of a CFT stress tensor in flat-space limit is zero, we avoid additional notation $\bar{T}_{\mu \nu}$.

\section{EFFECTIVE LIGHTCONE COMMUTATOR IN CFT}

We now discuss in what sense a lightcone limit is taken and what the dominating structure is. The tracelessness condition will be imposed. Let

$$
d s^{2}=-d x^{+} d x^{-}+\delta_{a b} d x^{a} d x^{b}
$$

where $\left(x^{+}, x^{-}\right)=(t+y, t-y)$. One has

$$
T^{ \pm \pm}=-2\left(T_{0}^{0} \mp T_{1}^{0}\right)-T_{a}^{a}, \quad T^{-+}=T_{a}^{a} .
$$

In $d=2, T^{++} / T^{--}$is independent of $x^{+} / x^{-}$, respectively, and $T_{a}^{a}=0$. Going beyond $d=2$, we consider the following lightcone limit:

$$
x^{-} \rightarrow 0 \quad \text { with } \quad x^{+} \text {fixed }
$$

(One can also consider $x^{+} \rightarrow 0$ with $x^{-}$fixed.) and focus on the effective commutation relation, where stress tensors are in a correlation function. The reason to adopt such a scenario is that, for many purposes, such as in the conformal block decomposition, stress tensors always appear in a correlator and thus having an effective commutator would be sufficient.

We shall focus on stress tensors with indices uncontracted, corresponding to the lowest-twist or largest-spin limit. Intuitively, the lightcone-component $T^{++}$should dominate near the lightcone. However, in $d>2$, the existence of $T_{a}^{a}$ in (22) causes trouble: since purely spatial components of the stress-tensor commutator do not have a model-independent expression, there is no universal way to compute $\left[T^{++}(x), T^{++}\left(x^{\prime}\right)\right]$ in $d>2$. This obstacle may be circumvented by adopting an effective lightcone commutator. Let us here demonstrate via an example. Consider the following CFT correlator [9]:

$$
\begin{gathered}
\left\langle T_{\mu \nu}\left(x_{1}\right) \phi\left(x_{2}\right) \phi(0)\right\rangle \sim a \frac{I_{\mu}^{\lambda}\left(x_{1}\right) I_{\nu}^{\sigma}\left(x_{1}\right) A_{\lambda \sigma}\left(x_{2}\right)}{x_{1}^{2 d} x_{2}^{2 \Delta_{\phi}+2-d}}, \\
I_{\mu}^{\lambda}(s)=\delta_{\mu}^{\lambda}-\frac{2 s^{\lambda} s_{\mu}}{s^{2}}, \quad A_{\lambda \sigma}(s)=s_{\lambda} s_{\sigma}-\frac{s^{2}}{d} \eta_{\lambda \sigma},
\end{gathered}
$$

where we focus on the short-distance (small $x_{2}$ ) behavior; $a$ is a constant and $\Delta_{\phi}$ the dimension of $\phi$. We will put scalar fields on a $x^{+}-x^{-}$plane and consider the lightcone limit $x^{-} \rightarrow 0$. The stress tensors generally allow the transversecoordinate dependence, and we consider that stress tensors also approach to the lightcone. One finds the following limiting behavior under (23):

$$
x_{1}^{2 d} x_{2}^{2 \Delta_{\phi}+2-d}\left\langle\left(T_{0}^{0}-T_{1}^{0}\right)\left(x_{1}\right) \phi\left(x_{2}\right) \phi(0)\right\rangle \sim\left(x_{2}^{+}\right)^{2},
$$

while having $T_{0}^{0}+T_{1}^{0}, T_{a}^{a}$, $T^{+a}$, or $T^{-a}$ in the correlator does not contribute in the same limit. Although this 
example involves only a stress tensor, one may consider more stress tensors and verify that the contributions near the lightcone are dominated by $\left(T_{0}^{0}-T_{1}^{0}\right)^{n}$ with $n$ stress tensors. We thus focus on the commutator involving $T_{0}^{0}-T_{1}^{0}$ as the effective lightcone description. In what follows, we let

$$
\tilde{T}^{++}=-2\left(T_{0}^{0}-T_{1}^{0}\right) .
$$

Let us also remark that the operator $\partial_{+} \tilde{T}^{++}$does not contribute in the same lightcone limit either.

We next compute the commutator of $\tilde{T}^{++}$using (9), (10), and (12), together with the tracelessness condition. We find, before imposing the lightcone limit, the following intermediate expression:

$$
\begin{aligned}
- & i\left[\tilde{T}^{++}(x), \tilde{T}^{++}\left(x^{\prime}\right)\right] \\
= & -4\left(\tilde{T}^{++}(x)+\tilde{T}^{++}\left(x^{\prime}\right)\right)\left(\partial_{+}-\partial_{-}\right) \delta^{d-1} \\
& +4\left(T_{a}^{a}(x)+T_{a}^{a}\left(x^{\prime}\right)\right)\left(\partial_{+}-\partial_{-}\right) \delta^{d-1} \\
& -4\left(T^{+a}(x)+T^{+a}\left(x^{\prime}\right)\right) \partial_{a} \delta^{d-1}+\tilde{s}\left(x, x^{\prime}\right),
\end{aligned}
$$

where $\delta^{d-1}=\delta\left(y-y^{\prime}\right) \delta^{d-2}\left(x^{a}-x^{\prime a}\right)$ and

$$
\begin{aligned}
\tilde{s}\left(x, x^{\prime}\right)= & 4\left(\partial_{\nu} t^{0 \nu, 00}\left(x, x^{\prime}\right)+\partial_{\nu} t^{0 \nu, 01}\left(x, x^{\prime}\right)\right. \\
& \left.-\partial_{\nu}^{\prime} t^{0 \nu, 01}\left(x^{\prime}, x\right)+\partial_{\nu} t^{1 \nu, 01}\left(x, x^{\prime}\right)\right)
\end{aligned}
$$

is the corresponding Schwinger term. At equal time, one may write the difference $\left(y-y^{\prime}\right)$ as either $\left(x^{+}-x^{\prime+}\right)$ or $-\left(x^{-}-x^{\prime}\right)$. We have here explicitly indicated the dimensionality of the delta function.

As a check on (28), let us take $d=2$, where $T_{a}^{a}=T^{+a}=\partial_{+} \tilde{T}^{++}=0$. We have

$$
\begin{aligned}
& -i\left[\tilde{T}^{++}\left(x^{-}\right), \tilde{T}^{++}\left(x^{\prime-}\right)\right] \\
& =4\left(\tilde{T}^{++}\left(x^{-}\right)+\tilde{T}^{++}\left(x^{\prime-}\right)\right) \partial_{-} \delta\left(x^{-}-x^{\prime-}\right)+\left.\tilde{s}\right|_{d=2},
\end{aligned}
$$

which is precisely the Virasoro algebra (1), as it must be, provided that the Schwinger term is related to the central charge via

$$
\left.\tilde{s}\right|_{d=2}=-\frac{2 c}{3 \pi} \partial_{-}^{3} \delta\left(x^{-}-x^{--}\right) .
$$

We leave the discussion on the Schwinger term to the next section.

In $d>2$, we shall focus on the lightcone limit where the additional $T_{a}^{a}, T^{+a}$ pieces in (28) are suppressed. The effective lightcone commutator may be written as

$$
\begin{aligned}
- & i\left[\tilde{T}^{++}\left(x^{+}, x^{a}\right), \tilde{T}^{++}\left(x^{\prime+}, x^{\prime a}\right)\right] \\
= & -4\left(\tilde{T}^{++}\left(x^{+}, x^{a}\right)+\tilde{T}^{++}\left(x^{\prime+}, x^{\prime a}\right)\right) \partial_{+} \delta^{d-1} \\
& +\tilde{s}\left(x^{+}, x^{a}, x^{\prime+}, x^{\prime a}\right),
\end{aligned}
$$

where $\delta^{d-1}=\delta\left(x^{+}-x^{\prime+}\right) \delta^{d-2}\left(x^{a}-x^{\prime a}\right)$. We have dropped the $x^{-}, x^{\prime-}$ dependence in the stress tensors and the Schwinger term to indicate that these contributions are localized on the lightcone. The lightcone limit is imposed after computing the commutation relation. In $d=2$, it is not necessary to impose a lightcone limit.

\section{REMARKS ON THE SCHWINGER TERM}

The connection between the Schwinger term and $C_{T}$ can be deduced from the spectral representation $[10,11]$. First, consider the Källen-Lehmann spectral form of the stresstensor two-point function in unitary QFT (in Euclidean signature),

$$
\begin{aligned}
& \left\langle T_{\mu \nu}(x) T_{\lambda \rho}(0)\right\rangle \\
& =N_{d} \int_{0}^{\infty} d \mu\left(\rho^{(0)}(\mu) \Pi_{\mu \nu, \lambda \rho}^{(0)}+\rho^{(2)}(\mu) \Pi_{\mu \nu, \lambda \rho}^{(2)}\right) G(x, \mu),
\end{aligned}
$$

where

$$
\begin{gathered}
N_{d}=\frac{2 \pi^{d / 2}}{(d-1)^{2}(d+1) 2^{d-1} \Gamma(d / 2)}, \\
\Pi_{\mu \nu, \lambda \rho}^{(0)}=\frac{1}{\Gamma(d)} S_{\mu \nu} S_{\lambda \rho}, \quad S_{\mu \nu}=\partial_{\mu} \partial_{\nu}-\delta_{\mu \nu} \partial^{2}, \\
\Pi_{\mu \nu, \lambda \rho}^{(2)}=\frac{d-1}{2 \Gamma(d-1)}\left(S_{\mu \lambda} S_{\nu \rho}+S_{\mu \rho} S_{\nu \lambda}-\frac{2 S_{\mu \nu} S_{\lambda \rho}}{d-1}\right), \\
G=\int \frac{d^{d} p}{(2 \pi)^{d}} \frac{e^{i p x}}{p^{2}+\mu^{2}}=\left(\frac{\mu}{2 \pi|x|}\right)^{\frac{d}{2}-1} \frac{K_{\frac{d}{2}-1}(\mu|x|)}{2 \pi} .
\end{gathered}
$$

The spectral functions $\rho^{(0)}(\mu), \rho^{(2)}(\mu)$ represent spin-0 and spin-2 intermediate states, respectively. Restricting to CFT, scale invariance implies

$$
\rho^{(0)}(\mu)=\rho^{(0)} \mu^{d-2} \delta(\mu), \quad \rho^{(2)}(\mu)=\rho^{(2)} \mu^{d-3} .
$$

These functions do not lead to an infrared singularity. $\rho^{(0)}(\mu)$ does not contribute in $d>2$ while $\Pi_{\mu \nu, \lambda \rho}^{(2)}$ vanishes in $d=2$. With (38), one can compute (33) and match with [12]

$$
\begin{aligned}
\left\langle T_{\mu \nu}(x) T_{\lambda \rho}(0)\right\rangle & =C_{T} \frac{\mathcal{I}_{\mu \nu, \lambda \rho}(x)}{x^{2 d}}, \\
\mathcal{I}_{\mu \nu, \lambda \rho}(x) & =\frac{1}{2}\left(I_{\mu \lambda}(x) I_{\nu \rho}(x)+I_{\mu \rho}(x) I_{\nu \lambda}(x)\right)-\frac{1}{d} \delta_{\mu \nu} \delta_{\lambda \rho},
\end{aligned}
$$

and find, in CFTs, 


$$
\rho^{(0)}=\frac{C_{T}}{2}, \quad \rho^{(2)}=\frac{d-1}{d} C_{T} .
$$

It is more involved to obtain the delta-function distribution from the Schwinger term, but it has been worked out a long time ago for unitary QFT $[13,14]$. As a concrete example in higher dimensions, we focus on $d=4$ in the following. The relevant results in $d=4$ Lorentzian CFT, where $\rho^{(0)}$ does not contribute and $\rho^{(2)}=\frac{3 C_{T}}{4}$, are

$$
\begin{aligned}
F_{\mu \nu, \lambda \rho}\left(x-x^{\prime}\right) \equiv & \left\langle\left[T_{\mu \nu}(x), T_{\lambda \rho}\left(x^{\prime}\right)\right]\right\rangle, \\
F_{00,00}= & F_{0 i, 0 j}=F_{00, i j}=0, \\
F_{00,0 i}= & -i N_{4} \int_{0}^{\Lambda} d \mu \rho^{(2)}(\mu) \partial_{i} \Delta \delta^{3}\left(x-x^{\prime}\right) \\
& -i \frac{N_{4}}{2} \rho^{(2)} \partial_{i} \Delta^{2} \delta^{3}\left(x-x^{\prime}\right) \\
= & -i \frac{C_{T} \pi^{2}}{480}\left(\Lambda^{2}+\Delta\right) \partial_{i} \Delta \delta^{3}\left(x-x^{\prime}\right),
\end{aligned}
$$

where $\Delta$ is a Laplacian. Only the equal-time commutators with an odd number of temporal indices are nonzero since the causal propagator is odd in time; $F_{0 i, j k}$ can be nonzero, but these components are irrelevant in the lightcone limit. Note that the fifth-derivative "boundary" term generally exists in $d=4$, unless one has restricted to field theories with $\rho^{(2)}(\mu \rightarrow \infty)=0$, which is however not a CFT.

In $d=2$, a similar analysis gives the following nonzero contribution:

$$
F_{00,01}=-i \frac{\pi C_{T}}{6} \partial_{1}^{3} \delta\left(x^{1}-x^{\prime 1}\right),
$$

where we have recalled (38) and (40), and this gives

$$
\left.\langle\tilde{s}\rangle\right|_{d=2}=-\frac{4 \pi C_{T}}{3} \partial_{-}^{3} \delta\left(x^{-}-x^{\prime-}\right),
$$

which reproduces the required identification (31) in $d=2$ CFT, with $C_{T}=\frac{c}{2 \pi^{2}}$ where $c=1$ for a free boson.

We remark that, a prior, the Schwinger term could be a $q$-number in general QFT. From the Virasoro algebra, one knows the corresponding Schwinger term must be a $c$-number in $d=2$ CFT with $\left.\tilde{s}\right|_{d=2}=\left.\langle\tilde{s}\rangle\right|_{d=2}$. For $d>2$, we shall here assume that we are restricted in the class of CFTs where the Schwinger term is a $c$-number, at least in the lightcone limit. A more general question whether the Schwinger term might always be a $c$-number goes beyond the scope of the present work and will not be addressed here. Note the Schwinger term must have a consistent dimension and the requirement that the $d \rightarrow 2$ limit of (28) reproduces the Virasoro algebra implies the Schwinger term in general $d$ should not touch the coefficient of the existing $\tilde{T}^{++}$piece. (One may adopt additional limits, such as a large $C_{T}$, to suppress possible unwanted contributions, but we do not consider such a limit here.)

On the other hand, a direct canonical computation shows that the Schwinger terms (14)-(17) in $d>2$ free theories simply vanish, as first pointed out in [4], while (18), which is however irrelevant in the lightcone limit, can be nonzero but its expression is model dependent. (Related computation details can be found in [15].) The authors of $[4,15]$ then argued that quantum effects are responsible for producing the anomalous $c$-number delta-function distribution predicted from the spectral forms. While their results suggest that the free theories belong to the class of theories we are interested in, it would be interesting to revisit the freetheory calculations and derive the $c$-number contribution in view of the results presented here.

In the class of $d=4$ CFTs, for instance, where the Schwinger term is effectively a $c$-number near the lightcone, we may write the lightcone effective algebra as

$$
\begin{aligned}
- & i\left[\tilde{T}^{++}\left(x^{+}, x^{a}\right), \tilde{T}^{++}\left(x^{\prime+}, x^{\prime a}\right)\right] \\
= & -4\left(\tilde{T}^{++}\left(x^{+}, x^{a}\right)+\tilde{T}^{++}\left(x^{\prime+}, x^{\prime a}\right)\right) \partial_{+} \delta^{3} \\
& +\frac{C_{T} \pi^{2}}{60}\left(\Lambda^{2}+\Delta\right) \partial_{+} \Delta \delta^{3},
\end{aligned}
$$

where $\delta^{3}=\delta\left(x^{+}-x^{\prime+}\right) \delta^{2}\left(x^{a}-x^{\prime a}\right)$. The appearance of the UV divergence in the Schwinger term is expected to be a general figure in $d>2$ CFTs, based on essentially dimensional analysis. The coefficient of the power-law divergence has no universal meaning and thus we shall focus on the universal finite piece (the coefficient of the highest-order derivative of the delta function) in the Schwinger term and subtract the divergence off when computing a correlator.

We hope to discuss the applications of the lightcone stress-tensor commutation relation elsewhere. In particular, it would be interesting to realize the lightcone algebra in a holographic framework and explore potential connections with the lowest-twist universality [8] and also some recent works [16-21].

\section{ACKNOWLEDGMENTS}

I thank L. Fitzpatrick, T. Hartman, C. Herzog, K. Jensen, H. Osborn, and A. Tseytlin for discussions and useful comments. This work was supported in part by the U.S. Department of Energy Office of Science under Award No. DE-SC0015845 and in part by the Simons Collaboration grant on the Nonperturbative Bootstrap. 
[1] M. Virasoro, Subsidiary conditions and ghosts in dualresonance models, Phys. Rev. D 1, 2933 (1970).

[2] J. Schwinger, Non-Abelian gauge fields. Relativistic invariance, Phys. Rev. 127, 324 (1962).

[3] J. Schwinger, Energy and momentum density in field theory, Phys. Rev. 130, 800 (1963).

[4] D. Boulware and S. Deser, Stress tensor commutators and Schwinger terms, J. Math. Phys. (N.Y.) 8, 1468 (1967).

[5] J. Maldacena, The large $\mathrm{N}$ limit of superconformal field theories and supergravity, Int. J. Theor. Phys. 38, 1113 (1999).

[6] E. Witten, Anti-de Sitter space and holography, Adv. Theor. Math. Phys. 2, 253 (1998).

[7] S. Gubser, I. Klebanov, and A. Polyakov, Gauge theory correlators from noncritical string theory, Phys. Lett. B 428, 105 (1998).

[8] A. Fitzpatrick and K.-W. Huang, Universal lowest-twist in CFTs from holography, J. High Energy Phys. 08 (2019) 138.

[9] J. Cardy, Anisotropic corrections to correlation functions in finite-size systems, Nucl. Phys. B290, 355 (1987).

[10] A. Cappelli, D. Friedan, and J. Latorre, C-theorem and spectral representation, Nucl. Phys B352, 616 (1991).

[11] H. Osborn and G. Shore, Correlation functions of the energy momentum tensor on spaces of constant curvature, Nucl. Phys. B571, 287 (2000).
[12] H. Osborn and A. Petkou, Implications of conformal invariance in field theories for general dimensions, Ann. Phys. (N.Y.) 231, 311 (1994).

[13] R. Brandt, Approach to equal-time commutators in quantum field theory, Phys. Rev. 166, 1795 (1968).

[14] K. Mahanthappa, Schwinger terms in stress tensor commutators, Phys. Rev. 181, 2087 (1969).

[15] J. Trubatch, Stress-tensor commutation relations, Nuovo Cimento A 68, 339 (1970).

[16] H. Casini, E. Teste, and G. Torroba, Modular Hamiltonians on the null plane and the Markov property of the vacuum state, J. Phys. A 50, 364001 (2017).

[17] N. Afkhami-Jeddi, T. Hartman, S. Kundu, and A. Tajdini, Shockwaves from the operator product expansion, J. High Energy Phys. 03 (2019) 201.

[18] C. Cordova and S. H. Shao, Light-ray operators and the BMS algebra, Phys. Rev. D 98, 125015 (2018).

[19] A. Belin, D. M. Hofman, and G. Mathys, Einstein gravity from ANEC correlators, arXiv:1904.05892.

[20] M. Kologlu, P. Kravchuk, D. Simmons-Duffin, and A. Zhiboedov, The light-ray OPE and conformal colliders, arXiv:1905.01311.

[21] S. Balakrishnan, V. Chandrasekaran, T. Faulkner, A. Levine, and A. Shahbazi-Moghaddam, Entropy variations and light ray operators from replica defects, arXiv:1906.08274. 